Bull. Austral. Math. Soc.

VOL. $63(2001) \quad[67-74]$

\title{
PERMUTATION POLYNOMIALS AND GROUP PERMUTATION POLYNOMIALS
}

\author{
Young Ho Park and June BoK LeE
}

\begin{abstract}
Permutation polynomials of the form $x^{\tau} f\left(x^{s}\right)$ over a finite field give rise to group permutation polynomials. We give a group theoretic criterion and some other criteria in terms of symmetric functions and power functions.
\end{abstract}

\section{INTRODUCTION}

Let $\mathbb{F}_{q}$ be a finite field of $q=p^{e}$ elements of characteristic $p$. A polynomial in $\mathbb{F}_{q}[x]$ is called a permutation polynomial over $\mathbb{F}_{q}$ if it is a bijection from $\mathbf{F}_{q}$ to $\mathbf{F}_{q}$. General study of permutation polynomials started with Hermite, followed by Dickson [3]. See [6] for general material about permutation polynomials, and $[4,5]$ for open problems concerning permutation polynomials, and [8] for recent results.

One of the families of permutation polynomials consists of polynomials of the form $x^{\tau} f\left(x^{s}\right)$, where $s \mid q-1$. This class originated from the work of Rogers and Dickson [3] who considered the case $f(x)=g(x)^{d}$, and then several other special cases have been studied by Carlitz and Wells [2], Niederreiter and Robinson [9]. Wan and Lidl [12] gave a simple unified treatment (criterion) for this class in terms of the primitive roots and determined its group structure. The purpose of this article is to give a group theoretic criterion for this family, and explain how this naturally leads to the notion of group permutation polynomials of a subgroup of the multiplicative group $G=\mathbb{F}_{q}^{*}$. Brison [1] also considered group permutation polynomials and generalised the Hermite criterion. In Section 3, we discuss a conjecture of Brison [1]. Turnwald [11] gave new criteria for permutation polynomials in terms of symmetric functions and power functions of their values. In the final section, we generalise these to group permutation polynomials.

\section{GROUP PERMUTATION POLYNOMIALS}

Let $N$ be a subgroup of the multiplicative group $G=\mathbb{F}_{q}^{*}$. A polynomial in $\mathbb{F}_{q}[x]$ is called a group permutation polynomial over $N$ or simply a permutation polynomial over

Received 27th March, 2000

This work is supported by BSRI-97-1423

Copyright Clearance Centre, Inc. Serial-fee code: 0004-9727/01 \$A2.00+0.00. 
$N$ if it induces a bijection on $N$. For example, if $(r,|N|)=1$ and $\alpha \in N, \alpha x^{r}$ is a group permutation polynomial over $N$. These are called monomials.

The permutation polynomials of the form $h(x)=x^{r} f\left(x^{s}\right)$ over $\mathbb{F}_{q}$ are closely related to the group permutation polynomials over some subgroup of $F_{q}^{*}$. As in [10], we may restrict our attention to polynomials $h(x)$ such that $(r, s)=1$ and $s \mid q-1$. Let $d=(q-1) / s$. Suppose that $h(x)=x^{r} f\left(x^{s}\right)$ is a permutation polynomial over $\mathbb{F}_{q}$. Since $f(x)$ has no nonzero roots, the group $G=\mathbb{F}_{q}^{*}$ is $f(x)$-stable. Let

$$
H=\left\{g \in G \mid g^{s}=1\right\}=\left\{g^{d} \mid g \in G\right\}
$$

and

$$
N=\left\{g^{s} \mid g \in G\right\}=\left\{g \in G \mid g^{d}=1\right\} .
$$

Note that $|H|=s$, and $|N|=d$.

Proposition 2.1. A polynomial $\phi(x)$ maps $N$ into $N$ if and only if $\phi(x) \equiv$ $x^{r} f(x)^{s}\left(\bmod x^{d}-1\right)$ for some $f \in \mathbb{F}_{q}[x]$.

Proof: Suppose $\phi(N) \subset N$. Let $\phi(x)=x^{r} \phi_{1}(x)$, where $\phi_{1}(0) \neq 0$. For each $a \in N$, $\phi_{1}(a) \in N$, and thus $\phi_{1}(a)=b_{a}^{s}$ for some $b_{a} \in G$. Choose a polynomial $f(x) \in \mathbb{F}_{q}[x]$ such that $f(a)=b_{a}$. Then $\phi(a)=a^{r} f(a)^{s}$ for all $a \in N$, and hence $\phi(x) \equiv x^{r} f(x)^{s}$ $\left(\bmod x^{d}-1\right)$. The converse is clear.

Proposition 2.2. For each $g \in G$, the restriction of $h(x)$ to the coset $g H$ is a bijection onto the coset $h(g) H$.

ProOF: For $\alpha \in H$, we have $h(g \alpha)=(g \alpha)^{r} f\left((g \alpha)^{s}\right)=\alpha^{r} h(g) \in h(g) H$. Thus $h(x)$ maps $g H$ into $h(g) H$. To prove that it is $1-1$, suppose $\alpha, \beta \in H$ and $h(g \alpha)=h(g \beta)$. As above, we then have $\alpha^{r} h(g)=\beta^{r} h(g)$, or $\left(\alpha \beta^{-1}\right)^{r}=1$. Since $\left(\alpha \beta^{-1}\right)^{s}=1$ and $(r, s)=1$, this implies that $\alpha=\beta$. Hence the restriction of $h(x)$ to $g H$ is an injection, and hence a bijection onto $h(g) H$.

By Proposition 2.2, $h(x)$ induces a well-defined map on $G / H$ given by

$$
\bar{h}: G / H \rightarrow G / H, \quad g H \mapsto h(g) H .
$$

We use the group isomorphism

$$
G / H \simeq N, \quad g H \mapsto g^{s}
$$

to transform $\bar{h}$ to a function $\phi_{h}$ on $N ; \phi_{h}\left(g^{s}\right)=h(g)^{s}=g^{r s} f\left(g^{s}\right)^{s}$. Hence $\phi_{h}$ is determined as

$$
\phi_{h}(x)=x^{r} f(x)^{s} .
$$

We can reverse our construction above. Suppose we are given a polynomial $\phi(x)=$ $x^{r} f(x)^{s}$ and a $\phi(x)$-stable subgroup $N$ of order $d$, where $d s=q-1$. Consider the 
polynomial $h(x)=x^{r} f\left(x^{s}\right)$. Then $G$ is $h(x)$-stable. Now it is clear, from the construction above, $h(x)$ is the unique polynomial of the given type such that $\phi_{h}=\phi$. We therefore have the following theorem.

THEOREM 2.3. $x^{\tau} f\left(x^{s}\right)$ is a group permutation polynomial over $G=\mathbf{F}_{q}^{*}$ if and only if $x^{r} f(x)^{s}$ is a group permutation polynomial over $N=\left\{g^{s} \mid g \in G\right\}$.

It is an easy matter to prove the following two well-known results [6] using Theorem 2.3.

Corollary 2.4. Let $(r, q-1)=1$. Then $h(x)=x^{r}\left(f\left(x^{s}\right)\right)^{(q-1) / s}$ is a permutation polynomial over $\mathbb{F}_{q}$ if and only if $f\left(x^{s}\right)$ has no root in $\mathbb{F}_{q}^{*}$.

Proof: $h(x)$ is a permutation polynomial over $F_{q}$ if and only if

$$
\phi(x)=x^{r}\left(f(x)^{(q-1) / s}\right)^{s}=x^{r} f(x)^{q-1}
$$

is a permutation polynomial over $N=\left\{g^{s} \mid g \in \mathbb{F}_{q}^{*}\right\}$ if and only if $f(x)$ has no root in $N$ if and only if $f\left(x^{s}\right)$ has no root in $\mathbb{F}_{q}^{*}$.

COROLLARY 2.5. $h(x)=x\left(x^{(q-1) / 2}+a\right)$ is a permutation polynomial over $\mathbf{F}_{q}$ if and only if $\left(a^{2}-1\right)^{(q-1) / 2}=1$.

Proof: $h(x)$ is a permutation polynomial over $\mathbb{F}_{q}$ if and only if $\phi(x)=x(x+a)^{(q-1) / 2}$ is a permutation polynomial over $\{ \pm 1\}$ if and only if $\phi(1) \phi(-1)=-\left(a^{2}-1\right)^{(q-1) / 2}=$ -1 .

In $[10]$, the authors examined permutation properties of the polynomials

$$
h_{k, r, s}(x)=x^{r}\left(1+x^{s}+\cdots+x^{s k}\right)
$$

over $\mathbb{F}_{q}$, where $k, r, s$ are positive integers. The study of these polynomials originated in [7]. Under suitable assumptions (see [10, Theorem 4.7]) it is proved, using the notion of circulant matrices, that if $h_{k, r, s}(x)$ is a permutation polynomial over $\mathbb{F}_{q}$, then $(k+1)^{s} \equiv$ $(-1)^{r-1}(\bmod p)$. Here we present a quick proof of this using Theorem 2.3. Suppose $h_{k, r, s}(x)$ is a permutation polynomial over $G=F_{q}^{*}$. By Theorem $2.3 \phi(x)=x^{r}(1+x+$ $\left.\cdots+x^{k}\right)^{s}$ is a permutation polynomial over $N=G^{s}$. Let $d=(q-1) / s$. As proved in $[10](k+1, d)=1$ so that $x^{k+1}$ permutes $N$ and $N-\{1\}$. We thus have, in $\mathbb{F}_{q}^{*}$,

$$
\begin{aligned}
(-1)^{d-1}=\prod_{a \in N} a=\prod_{a \in N} \phi(a) & =(k+1)^{s} \prod_{1 \neq a \in N} a^{r}\left(\frac{1-a^{k+1}}{1-a}\right)^{s} \\
& =(k+1)^{s}(-1)^{(d-1) r}\left(\frac{\prod_{a \neq 1}\left(1-a^{k+1}\right)}{\prod_{a \neq 1}(1-a)}\right)^{s} \\
& =(k+1)^{s}(-1)^{(d-1) r}\left(\frac{\prod_{a \neq 1}(1-a)}{\prod_{a \neq 1}(1-a)}\right)^{s} \\
& =(k+1)^{s}(-1)^{(d-1) r} .
\end{aligned}
$$

Therefore, we have $(k+1)^{s} \equiv(-1)^{(d-1)(r-1)} \equiv(-1)^{r-1}(\bmod p)$. 


\section{H-UNIFORMITY}

Let $\omega$ be the primitive element of the multiplicative group $G=F_{q}^{*}$ so that $G=\langle\omega\rangle$, and let $H$ be a subgroup of order $s$ of $G$. Let $d=(q-1) / s$. Then $H=\left\langle\omega^{d}\right\rangle$ and

$$
G=H \cup H \omega \cup \cdots \cup H \omega^{d-1} .
$$

Let $P(G)$ be the group of permutation polynomials over $G=\mathbb{F}_{q}^{*}$ and let $P(G / H)$ be the subgroup of $P(G)$ consisting of permutation polynomials of $G$ which induces a permutation of $G / H$.

Observe that $f \in P(G / H)$ if and only if there is a permutation $\pi$ in $S_{d}$, the symmetric group on $\{0,1, \ldots, d-1\}$, and permutation polynomials $f_{0}, \ldots, f_{d-1}$ of $H$ such that

$$
f: h \omega^{i} \mapsto f_{i}(h) \omega^{\pi(i)}
$$

for all $h \in H$ and $0 \leqslant i \leqslant d-1$. If all $f_{i}(x) \in P(H)$ are monomials of degree $r$ with $(r, s)=1$, then $f$ is called an $H$-uniform permutation of $G$ of index $r[1]$. An $f \in P(G)$ is called an $H$-uniform polynomial of index $r$ if $f(x)$ is of the form

$$
f(x)=x^{r}\left(a_{0}+a_{1} x^{s}+\cdots+a_{d-1} x^{(d-1) s}\right)
$$

with $a_{i} \in \mathbb{F}_{q}[\mathbf{1}]$.

The following two results are proved in [1].

ThEOREM 3.1. Let $\pi \in S_{d}, f_{i} \in P(H), 0 \leqslant i \leqslant d-1$ where

$$
f_{i}(x)=a_{i, 1} x+\cdots+a_{i, s-1} x^{s-1} .
$$

Then there exists a unique permutation polynomial $f \in P(G / H)$ of degree $\leqslant q-2$ such that

1. the coefficients of $x^{s}, x^{2 s}, \ldots, x^{(d-1) s}$ are all zero;

2. $f\left(h \omega^{i}\right)=f_{i}(h) \omega^{\pi(i)}$ for all $h \in H$ and $0 \leqslant i \leqslant d-1$;

3. if there exists $j$ such that $a_{i, j}=0$ for all $i$, then the coefficients in $f$ of $x^{j}, x^{s+j}, \ldots, x^{(d-1) s+j}$ are all zero.

COROLlary 3.2. If $(r, s)=1$, and $\alpha_{0}, \ldots, \alpha_{d-1} \in H$, then there exists a polynomial of the form $f(x)=x^{r}\left(a_{0}+a_{1} x^{s}+\cdots+a_{d-1} x^{(d-1) s}\right)$ in $P(G)$ such that $f\left(h \omega^{i}\right)=\alpha_{i} h^{r} \omega^{\pi(i)}$ for all $i$. That is, every $H$-uniform permutation is induced by a suitable $H$-uniform polynomial.

Brison [1] considered a pair of finite subgroups $H \leqslant G \leqslant K^{*}$ inside any field $K$ and has conjectured that every $H$-uniform polynomial is a $H$-uniform permutation and proved it in several cases. Even in this general setting, the following argument shows that his conjecture is true. Let $H$ be a subgroup of a finite subgroup $G=\langle\omega\rangle$ of $K^{*}$ and suppose that

$$
f(x)=x^{r}\left(a_{0}+a_{1} x^{s}+\cdots+a_{d-1} x^{(d-1) s}\right)
$$


is a $H$-uniform polynomial, where $s=|H|$. For any $a, b \in G$ with $a^{s}=b^{s}$, we have

$$
\begin{aligned}
f(a)^{s} & =a^{r s}\left(a_{0}+a_{1} a^{s}+\cdots+a_{d-1} a^{(d-1) s}\right)^{s} \\
& =b^{r s}\left(a_{0}+a_{1} b^{s}+\cdots+a_{d-1} b^{(d-1) s}\right)^{s} \\
& =f(b)^{s} .
\end{aligned}
$$

Thus $f$ induces a permutation on $G / H$, that is, $f \in P(G / H)$. As before, we have

$$
f\left(h \omega^{i}\right)=f_{i}(h) \omega^{\pi(i)}
$$

for some $f_{0}, \ldots, f_{d-1} \in P(H)$ and $\pi \in S_{d}$, where $d=|G / H|$. In particular,

$$
\omega^{i r}\left(a_{0}+a_{1} \omega^{i s}+\cdots+a_{d-1} \omega^{i(d-1) s}\right)=f\left(\omega^{i}\right)=f_{i}(1) \omega^{\pi(i)} \text {. }
$$

Thus we have

$$
f\left(h \omega^{i}\right)=h^{r} \omega^{i r}\left(a_{0}+a_{1} \omega^{i s}+\cdots+a_{d-1} \omega^{i(d-1) s}\right)=h^{r} f_{i}(1) \omega^{\pi(i)}
$$

Therefore, we have:

TheOREM 3.3. $f \in P(G)$ is an $H$-uniform permutation if and only if it is an $H$-uniform polynomial.

\section{NEW CRITERIA FOR GROUP PERMUTATION POLYNOMIALS}

Let $H$ be a subgroup of order $s$ of $\mathbb{F}_{q}^{*}$. The following generalised version of the Hermite criterion for group permutation polynomials is proved in [1].

THEOREM 4.1. For $f(x) \in \mathbb{F}_{q}[x]$, let

$$
f(x)^{t}=q_{t}(x)\left(x^{s}-1\right)+f_{t}(x), \quad \operatorname{deg}\left(f_{t}\right)<s,
$$

and let $f_{t}(0)$ be the constant term of $f_{t}(x)$. Then $f(x)$ induces a permutation on $H$ if and only if

1. $f_{s}(x)=1$,

2. $f_{t}(0)=0$ for each $1 \leqslant t \leqslant s-1$.

For $f \in \mathbb{F}_{q}[x]$ of degree $\leqslant s$, we shall define, following Turnwald [11], three quantities $u, w, v$ and investigate their properties. First define the symmetric polynomials $S_{k}(f)$ on the values of $f$ by the equation

$$
\prod_{a \in H}(x-f(a))=\sum_{k=0}^{s}(-1)^{k} S_{k}(f) x^{s-k} .
$$


Let $u=u(f)$ be the smallest positive integer $k$ such that $S_{k}(f) \neq 0$ if such $k$ exists and otherwise set $u=\infty$. It is easy to see that $u=s-\operatorname{deg}\left(x^{s}-\prod_{a \in B}(x-f(a))\right)$. Next let

$$
P_{k}(f)=\sum_{a \in B} f(a)^{k}
$$

and define $w=w(f)$ to be the smallest positive integer $k$ such that $P_{k}(f) \neq 0$ if such $k$ exists, otherwise set $w=\infty$. Replacing $f(x)$ by $f(x)^{k}\left(\bmod x^{s}-1\right)$, we see that $w$ is the smallest positive integer such that $f(x)^{k}\left(\bmod x^{s}-1\right)$ has a nonzero constant term. Finally let

$$
v=v(f)=|f(H) \cap H| .
$$

THEOREM 4.2. If $f$ is a group permutation polynomial over $H$, then $u=w=$ $v=s$.

Proof: Since a permutation polynomial $g(x)$ of $H$ permutes the elements of $H$, we have $u(f)=u(f \circ g), w(f)=w(f \circ g)$, and $v(f)=(f \circ g)$. Thus it suffices to prove the statement for $f(x)=x$. Suppose $f(x)=x$. Since $H$ is the set of roots of $x^{s}-1$, we have

$$
\prod_{a \in B}(x-f(a))=\prod_{a \in H}(x-a)=x^{s}-1,
$$

and hence $u(f)=s$. Also $P_{k}(f)=\sum_{a \in H} f(a)^{k}=\sum_{a \in H} a^{k} \neq 0$ if and only if $k \equiv 0(\bmod s)$. Thus $w(f)=s$. Finally it is clear that $v(f)=s$.

THEOREM 4.3. If $w<\infty$, then $w \leqslant v$.

Proof: Let $g(x)=d \sum_{a \in H}(x-f(a))^{q-1}+x^{q-1}$. Since

$$
\begin{aligned}
(x-f(a))^{q-1} & =\frac{(x-f(a))^{q}}{x-f(a)}=\frac{x^{q}-f(a)^{q}}{x-f(a)} \\
& =x^{q-1}+x^{q-2} f(a)+\cdots+x f(a)^{q-2}+f(a)^{q-1}
\end{aligned}
$$

we have

$$
\begin{aligned}
g(x) & =d\left(s x^{q-1}+P_{1}(f) x^{q-2}+\cdots+P_{q-2}(f) x+P_{q-1}(f)\right)+x^{q-1} \\
& =d\left(P_{1}(f) x^{q-2}+\cdots+P_{q-2}(f) x+P_{q-1}(f)\right) .
\end{aligned}
$$

Therefore $\operatorname{deg} g=q-1-w$. For each $b \in \mathbb{F}_{q}^{*}$, let $n_{b}=|\{a \in H \mid f(a)=b\}|$. Then $g(b)=d \sum_{a \in H}(b-f(a))^{q-1}+1=d\left(s-n_{b}\right)+1=-d n_{b}$. In particular, if $0 \neq b \notin f(H)$, then $g(b)=0$. Thus $\operatorname{deg} g \geqslant q-1-v$. Since $\operatorname{deg} g=q-1-w$, we conclude that $w \leqslant v$.

THEOREM 4.4. If $f(H) \subsetneq H$, then $v+u \leqslant s$. 
Proof: Consider the polynomial $g(x)=x^{s}-1-\prod_{a \in H}(x-f(a))$. Note $f(x)$ is a permutation polynomial of $H$ if and only if $g(x)=0$. Since $g(f(b))=0$ for all $b \in H$, we have $v \leqslant \operatorname{deg} g$. But $g(x)=S_{1}(f) x^{s-1}-S_{2}(f) x^{s-2}+\cdots+(-1)^{s+1} S_{s}(f)-1$. Thus $\operatorname{deg} g=s-u$. Hence $v \leqslant s-u$.

THEOREM 4.5. If $f \neq 0$ and $w<\infty$, then $u \leqslant w$.

Proof: By Newton's formula, for any $k \geqslant 1$ we have

$$
P_{k}=S_{1} P_{k-1}-S_{2} P_{k-2}+\cdots+(-1)^{k-2} S_{k-1} P_{1}+(-1)^{k-1} k S_{k} .
$$

In particular, $P_{w}=(-1)^{w-1} w S_{w}$. Thus it suffices to show that $p$ does not divide $w$. But if $w=p j$, then

$$
0 \neq P_{w}=\sum_{a \in H} f(a)^{p j}=\left(\sum_{a \in B} f(a)^{j}\right)^{p}
$$

and hence $P_{j} \neq 0$, a contradiction.

THEOREM 4.6. If $f(0)=0$, then $u \geqslant s /(\operatorname{deg} f)$.

Proof: Let $n=\operatorname{deg} f$ and suppose $1<k<s / n$. Then

$$
\operatorname{deg} S_{k}\left(f\left(x_{1}\right), \ldots, f\left(x_{s}\right)\right) \leqslant n k<s .
$$

By the fundamental theorem of symmetric polynomials, we have

$$
S_{k}\left(f\left(x_{1}\right), \ldots, f\left(x_{s}\right)\right)=P\left(S_{1}\left(x_{1}, \ldots, x_{s}\right), \cdots, S_{s-1}\left(x_{1}, \ldots, x_{s}\right)\right)
$$

for some polynomial $P$ and the constant term of $P$ is obtained when $x_{1}=\cdots=x_{s}=0$. Since $f(0)=0$, this constant term is 0 . Now let $H=\left\{a_{1}, \ldots, a_{s}\right\}$, so that

$$
S_{k}\left(f\left(a_{1}\right), \ldots, f\left(a_{s}\right)\right)=P\left(S_{1}\left(a_{1}, \ldots, a_{s}\right), \ldots, S_{s-1}\left(a_{1}, \ldots, a_{s-1}\right)\right) .
$$

But $x^{s}-1=\prod_{i=1}^{s}\left(x-a_{i}\right)=x^{s}-S_{1} x^{s-1}+S_{2} x^{s-2}-\cdots+(-1)^{s-1} S_{s-1} x-1$. Thus $S_{i}\left(a_{1}, \ldots, a_{s}\right)=0$ for all $i=1, \ldots, s-1$. Therefore

$$
S_{i}\left(f\left(a_{1}\right), \ldots, f\left(a_{s}\right)\right)=P(0, \ldots, 0)=0 .
$$

Consequently, if $1<k<s / n$, then $S_{k}(f)=0$. Hence $u \geqslant s / n$.

COROLLARY 4.7. If $f(0)=0$ and $f(H) \varsubsetneqq H$, then $v \leqslant s-s /(\operatorname{deg} f)$.

THEOREM 4.8. Let $f(H) \subset H, f(0)=0$ and $\operatorname{deg} f=n$. Then the following statements are equivalent:

1. $f$ is a group permutation polynomial over $H$;

2. $u=s$; 

3. $w=s$;
4. $v=s$;
5. $v>s-(s / n)$;
6. $u>s-v$;
7. $u>(s / 2)$;
8. $s-u<w$.

Proof: Clearly (1) implies all. Note that $w<\infty$ since $f(H) \subset H$. By Theorem 4.5, (2) implies (3). By Theorem 4.3, (3) implies (4). Clearly (4) implies (1). By Corollary 4.7, (5) implies (1). Finally (6) implies (1) by Theorem 4.4 .

\section{REFERENCES}

[1] O.J. Brison, 'On group-permutation polynomials', Portugal Math. 59 (1993), 335-383.

[2] L. Carlitz and C. Wells, 'The number of solutions of a special system of equations in a finite field', Acta Arith. 12 (1966), 77-84.

[3] L.E. Dickson, Linear Groups with an exposition of the Galois field theory (Dover, New York, 1958).

[4] R. Lidl and G.L. Mullen, 'When does a polynomial over a finite field permute the elements of the field?', Amer. Math. Monthly 100 (1988), 243-246.

[5] R. Lidl and G.L. Mullen, 'When does a polynomial over a finite field permute the elements of the field? II', Amer. Math. Monthly 95 (1993), 71-74.

[6] R. Lidl and H. Niederreiter, Finite fields, Encyclopedia Math. Appl. 20 (Addison-Wesley, Reading, MA, 1983).

[7] R. Matthews, 'Permutation properties of the polynomials over a finite field', Proc. Amer. Math. Soc. 120 (1994), 47-51.

[8] G.L. Mullen, 'Permutation polynomials: a matrix analog of Schur's conjecture and a survey of recent results', Finite Fields Appl. 1 (1995), 242-258.

[9] N. Niederreiter and K.H. Robinson, 'Complete mappings of finite fields', J. Austral. Math. Soc. 33 (1982), 197-212.

[10] Y.H. Park and J.B. Lee, 'Permutation polynomials with exponents in an arithmetic progression', Bull. Austral. Math. Soc. 57 (1998), 243-252.

[11] G. Turnwald, 'A new criterion for permutation polynomials', Finite Fields Appl. 1 (1995), 64-82.

[12] D. Wan and R. Lidl, 'Permutation polynomials of the form $x^{r} f\left(x^{(q-1) / d}\right)$ and their group structures', Montash. Math. 112 (1991), 149-163.

Department of Mathematics

Kangwon National University

Chuncheon 200-701

Korea

e-mail: yhpark@math.kangwon.ac.kr
Department of Mathematics

Yonsei University

Seoul 120-749

Korea

e-mail: leejb@bubble.yonsei.ac.kr 